\title{
Towards Cable Parallel Robot Simulation for Control End Effector Position
}

\author{
Fouad INEL \\ Mechanical engineering department, Automatic Laboratory \\ University of Skikda \\ Skikda, Algeria \\ Email: f.innel [AT] univ-skida.dz
}

\author{
Ali MEDJBOURI \\ Mechanical engineering department, Automatic Laboratory \\ University of Skikda \\ Skikda, Algeria \\ Email: Ali.medjbouri [AT] gmail.com
}

\begin{abstract}
In this paper, we present a Graphical User Interface (GUI) simulator that has been developed using robust generalize predictive control. The proposed control technique is used for dealing with linear system uncertainties. The main contribution of this work is firstly: a graphical user interface has been developed and implemented based on geometric model, in order to, control the position of the end effector with several spatial tests. Secondly, we study the response of differential equations for our system with the proposed control for different trajectories in order to test the accurate tracking of the robot for desired trajectory simulation using MATLAB/Simulink. The Simulation results are carried out to prove the feasibility and effectiveness of the strategy outlined here.
\end{abstract}

Keywords- Cable driven robot, Robustness, generalized predictive control, GUI.

\section{INTRODUCTION}

Cable parallel robots are parallel robots where the endeffector is linked to the base platform by replacing of cable lengths and tensions permit the displacement and the application of efforts on the platform [1,2]. These robots have few moving parts, with reduced mass, and are most suitable for tasks requiring high performance such as speed and accuracy and provide a large workspace [3].The bestknown application is the Skycam, a camera controlled by a cables mechanism that is used for tele-diffusion of professional football games [4]. Another area of interest in biomedical applications such as tracking the movement of body parzts. An example is the Cassino Tracking System (CaTraSys) was used for the identification of kinematic parameters and the mobility of man $[5,6]$.

These offer incontestable advantages in comparison to the robots of classic architecture, this last it is a type of parallel manipulator used as a transmission way of cables connects a fixe base with a mobile platform. The coordinate controller One of the key aspects for cable driven robots is the need of a proper control strategy to achieve proper motions without breaking the cables. The Model Predictive method has been designed to improve the robustness of robotic system control, as reported for example in [7, 8]. In particular, a Predictive controller can adjust the control torque based on real-time position tracking error in the set-point control of the end-effector. The aim of this work is to control the position of end effector for different tests. Furthermore, present a simulation result of this $3 \mathrm{D}$ robot permitting to digitalize the appearance of it with generalize predictive control.

\section{SYSTEM STRUCTURE}

Figure 1 shows our virtual prototype robot with four cables. The base is fixed and each cable is attached to the one end of the platform. As a result of motors moments, cables wrap around the pulley to control the position and the orientation of the end-effector. The four cables-based robot allow a 3D plan movement with 4 degrees of freedom.

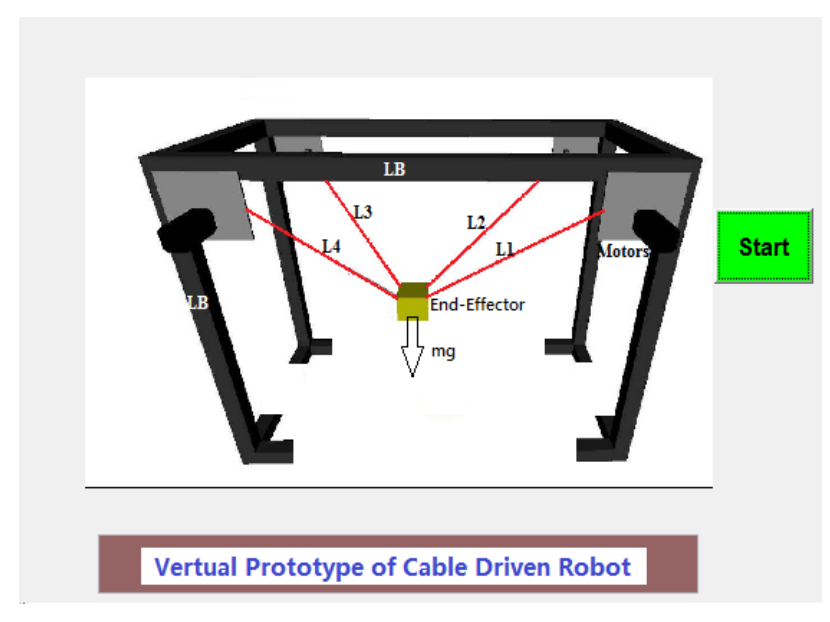

Figure 1. A virtual prototype of 3D parallel driven robot 


\section{GEOMETRIC MODELING}

In this section, we present the direct and inverse geometric model for our 3D cable-driven robots.

\section{A. Inverse Geometric Model (IGM)}

The aims of this section, is to determine the lengths of the cables "Li", the angles " $\theta \mathrm{i}$ " between the $\mathrm{X}, \mathrm{Y}$ axes and the cables connected to the mobile platform and " $\alpha i$ " between the $\mathrm{Z}$ axe the plane $\mathrm{X}, \mathrm{Y}$. The inverse geometric model can be expressed by the following equations [9].

$$
\begin{gathered}
L i=\sqrt{(x-A i x)^{2}+(y-A i y)^{2}+(z-A i z)^{2}} \\
\Theta i=\arctan g\left(\frac{y-A i y}{x-A i x}\right) \\
\alpha i=\arctan g\left(\frac{z-A i z}{\sqrt{(x-A i x)^{2}+(y-A i y)^{2}}}\right.
\end{gathered}
$$

\section{DyNAMIC MODEL OF THE END EFFECTOR}

In this section, we begin by presenting the dynamic equation of the robot with four cables. Then, the response will be simulated in closed loop with the generalize predictive controller. The dynamic model of the actuator is expressed by the following relationship [10]:

$$
m \ddot{X}=F_{R}
$$

Where: $\mathrm{m}$ :is the mass matrix.

$\ddot{X}$ :is the acceleration vector of the end-effector.

$F_{R}=\left(\begin{array}{lll}F_{R x} & F_{R y} & F_{R z}\end{array}\right)^{T}:$ is the resultant force of the all tensions applied to the cables.

$$
\left(\begin{array}{ccc}
m & 0 & 0 \\
0 & m & 0 \\
0 & 0 & m
\end{array}\right)\left(\begin{array}{c}
\ddot{x} \\
\ddot{y} \\
\ddot{z}
\end{array}\right)=\left(\begin{array}{l}
F_{R x} \\
F_{R y} \\
F_{R z}
\end{array}\right)
$$

\section{B. The Dynamic Comportment of the Motors}

The dynamic comportment of the motor is represented in Figure 2 and its expression is expressed by the following equation.

$$
J \ddot{\beta}+C \dot{\beta}=\tau-r T .
$$

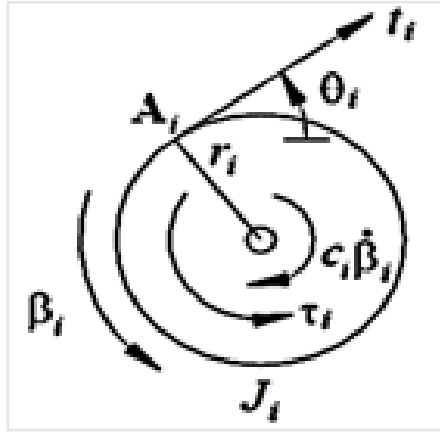

Figure 2. Structure pulley

with:

$$
\begin{aligned}
\text { Jmat } & =\left(\begin{array}{cccc}
J_{1} & 0 & 0 & 0 \\
0 & J_{2} & 0 & 0 \\
0 & 0 & J_{3} & 0 \\
0 & 0 & 0 & J_{4}
\end{array}\right) \\
\text { Cmat } & =\left(\begin{array}{cccc}
C_{1} & 0 & 0 & 0 \\
0 & C_{2} & 0 & 0 \\
0 & 0 & C_{3} & 0 \\
0 & 0 & 0 & C_{4}
\end{array}\right)
\end{aligned}
$$

We consider that all the rays of the pulley are the same: $r_{i}(i=1, \ldots, 4)=r=1 \quad \mathrm{~cm}$

$\tau\left(\tau_{1} . \tau_{2}, \ldots \tau_{i}\right)^{T}$ is the vector of the torques applied by the motors.

$\mathrm{t}(\mathrm{t} 1, \mathrm{t} 2, \ldots \mathrm{ti})^{\mathrm{T}}$ : is the vector of tension cables.

$\beta$ :is the angle of rotation of the pulley.

$\Theta i$ :The angles between cables and the pulley. So:

$$
t=\frac{1}{r}(\tau-J \ddot{\beta}-C \dot{\beta})
$$

Where LiO are the initial lengths of the cables:

$$
L_{i 0}=\sqrt{(A i x)^{2}+(A i y)^{2}+(A i z)^{2}}
$$

So

$$
\beta=\left(\begin{array}{l}
\beta_{1}(X) \\
\beta_{2}(X) \\
\vdots \\
\beta i(X)
\end{array}\right)=\frac{1}{r}\left(\begin{array}{l}
L_{10}-L_{1} \\
L_{20}-L_{2} \\
\vdots \\
L_{i 0}-L_{i}
\end{array}\right)
$$


by subtracting successively (9) with respect to time, we get:

$$
\ddot{\beta}=\frac{d}{d t}\left(\frac{\partial \beta}{\partial x}\right) \dot{x}+\frac{\partial \beta}{\partial x} \ddot{x}
$$

Finally, the set of equations of the dynamic model can be expressed in a standard form for robotic systems (11):

$$
\begin{aligned}
& \ddot{X}(t)=M^{-1}(X) * N(X, \dot{X})+M^{-1}(X) * m g(x)+ \\
& M^{-1}(X) * S(X) * \tau
\end{aligned}
$$

$\mathrm{M}(\mathrm{x})$ : Mass matrix of the system, $\mathrm{g}(\mathrm{x})$ : Vector of gravity terms and $\mathrm{m}$ : is the mass of end effector.

\section{PROPOSED CONTROL}

This section, presents our control demonstration, which has been ensured by means of the implementation. This proposed controller based on the overall system Cartesian dynamics equations of motion (Equation 11) using a Matlab program simulation to achieve reasonable performance for the trajectories.

Consider the following MIMO nonlinear system:

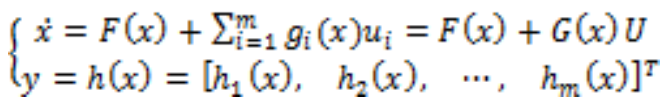

where $x \in \mathrm{Rn}, \mathrm{U} \in \mathrm{Rm}$ and y $€ \mathrm{Rm}$ are, respectively, the state vector, the control vector and the output vector. $\mathrm{F}(\mathrm{x})$ and $\mathrm{h}(\mathrm{x})$ are smooth vector fields[11, 12].

Generalize predictive controller is designed such that the future output y $(\mathrm{t}+\mathrm{T})$ follows the future reference signal $\mathrm{yr}$ $(\mathrm{t}+\mathrm{T})$ minimizing the quadratic performance index given below:

$$
l=\int_{0}^{T} e(t+\tau)^{T} e(t+\tau) d \tau
$$

Where: $e(t+T)$ is the future tracking error vector, and $T>$ 0 is the prediction horizon.

The outputs of the system and the reference signals predictions are approximated by their Taylor-series expansions up to corresponding relative degree $\rho_{i}$ :

$$
\begin{array}{r}
e_{i}(t+T) \approx \sum_{k=0}^{\rho_{i}} \frac{T^{k}}{k !}\left(y_{r_{i}}^{(\mathbb{C k})}(t)-y_{i}^{(\mathrm{Ck})}(t)\right) \\
e_{i}(t+T) \approx\left[\begin{array}{lllll}
1 & T & \frac{T}{2 !} & \cdots & \frac{T^{\rho_{i}}}{\rho_{i} !}
\end{array}\right]\left[\begin{array}{cc}
y_{r_{i}}(t)- & y_{i}(t) \\
\dot{y}_{r_{i}}(t)- & \dot{y}_{i}(t) \\
\ddot{y}_{r_{i}}(t) & \ddot{y}_{i}(t) \\
\vdots & \vdots \\
\ddot{y}_{r_{i}}^{\left(\rho_{i}\right)}(t) & y_{i}^{\left(\rho_{i}\right)}(t)
\end{array}\right]
\end{array}
$$

$$
e_{\mathrm{i}}(t+T)=\Gamma_{\mathrm{i}}(T) Y_{\mathrm{i}}(t)
$$

The outputs yi time derivatives required in the approximated performance index are expressed as given below using Lie derivatives:

$$
\left\{\begin{array}{c}
\dot{y}=L_{F} h_{1}(x) \\
\ddot{y}=L_{F}^{2} h_{1}(x) \\
\vdots \\
y_{1}^{\left(\rho_{1}\right)}=L_{F}^{\left(\rho_{1}\right)} h_{1}(x)+L_{g_{1}} L_{F}^{\left(\rho_{1}-1\right)} h_{1}(x) u_{1}+\cdots+L_{g_{m}} L_{F}^{\left(\rho_{1}-1\right)} h_{1}(x) u_{m} \\
\dot{y}_{2}=L_{F} h_{2}(x) \\
\vdots \\
\vdots \\
y_{1}^{\left(\rho_{m}\right)}=L_{F}^{\left(\rho_{m}\right)} h_{m}(x)+L_{g_{1}} L_{F}^{\left(\rho_{m}-1\right)} h_{m}(x) u_{1}+\cdots+L_{g_{m}} L_{F}^{\left(\rho_{m}-1\right)} h_{m}(x) u_{m}
\end{array}\right.
$$

Introducing (18) in the approximated performance index

$$
\text { (16) yields: }
$$

$$
J \approx(A(x)-B(x, U))^{T} \Pi(T)(A(x)-B(x, U))
$$

Finally, the control law minimizing the approximated performance index is obtained by solving the system:

$$
\frac{d I}{d U}=\left(\frac{d(A(x)-E(x, U))}{d U}\right)^{T} \Pi(T)(A(x)-B(x, U))=0_{(\rho p+m) \times 1}
$$

Consequently, the control law is given by [12]:

$$
\begin{aligned}
U & =D^{-1}(x) \operatorname{Diag}\left(\left(\Pi_{i}^{s s}\right)^{-1} \Pi_{i}^{s}\right) A(x) \\
& =D^{-1}(x) \operatorname{Diag}\left(\left(k_{i}\right) A(x)\right.
\end{aligned}
$$

The control architecture as shown in Figure 3 is made up of three different parts: the generalize predictive controller, the tension calculation and pulley angle $\beta$ to determine the cable lengths Li.

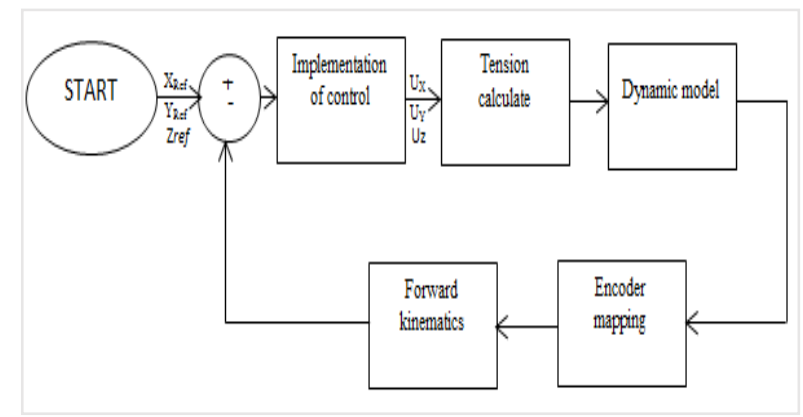

Control Architecture 


\section{RESULTS THE SIMULATION}

which has a non-linear equation system, for this purpose, we use a Runge Kutta method as a numeric solution and then we implement a Cartesian generalize predictive controller in this dynamic equation for reduce the tracking error $e x=\left(X_{\text {desired }}-X_{\text {actual }}\right)$. parameters for the dynamics equations of motion (11) for four cables are: point mass $\mathrm{m}=0.01 \mathrm{~kg}$; rotational shaft/pulley inertias $J_{i}=(i=1, \ldots, 4)=0.0008 \mathrm{Kgm}^{2}$. shaft rotational viscous damping

$$
C_{i}=(i=1, \ldots, 4)=0.01 \mathrm{Nms}
$$

$r_{i}(i=1, \ldots, 4)=r=1 \mathrm{~cm}$. So we put the reference of our system in the center of the workspace $(0,0,0)$. Figure 4,5 , 6,7 shows a graphical user interface of implementation the point-to-point command. i.e the user of this interface can enter the coordinates of any point into the workspace, when clicked on the plot, the end effector displaced directly to this point with a high precision, and also, this interface can initialization the case of this robot (Figure 7), these technique based on inverse geometric model.

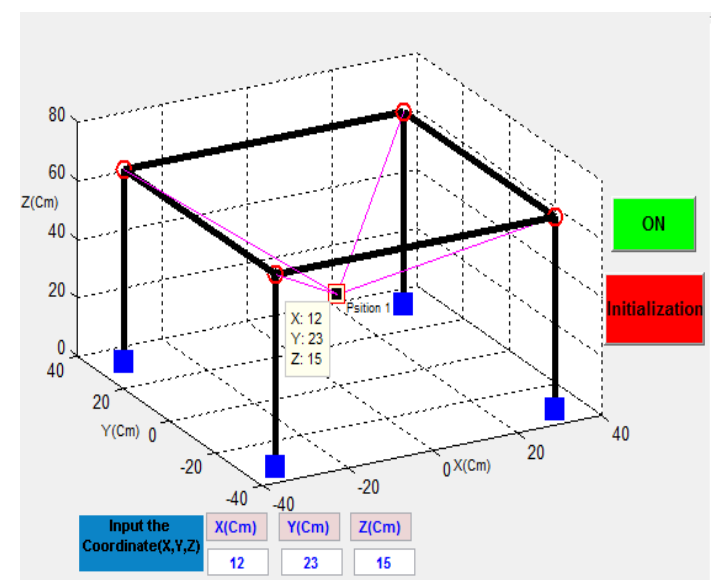

Figure 3. Plot the displacement of end effector (position 1)

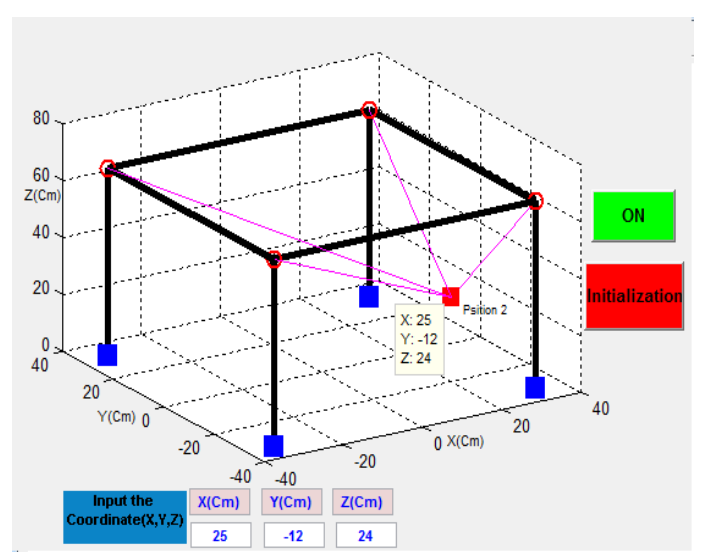

Figure 4. Plot the displacement of end effector (position 2)

For illustrate the role of our robust control, we use another high level graphical user interface command according to the object's colors, when, we have clicked on the color object, the end effector move and displace to this target object in workspace, this simulation technique based on the learning method.

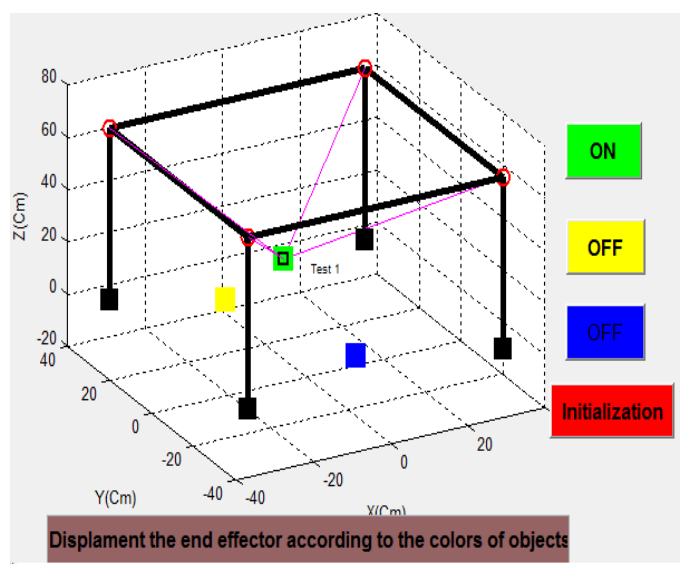

Figure 5. Displacement the end effector according to the green color

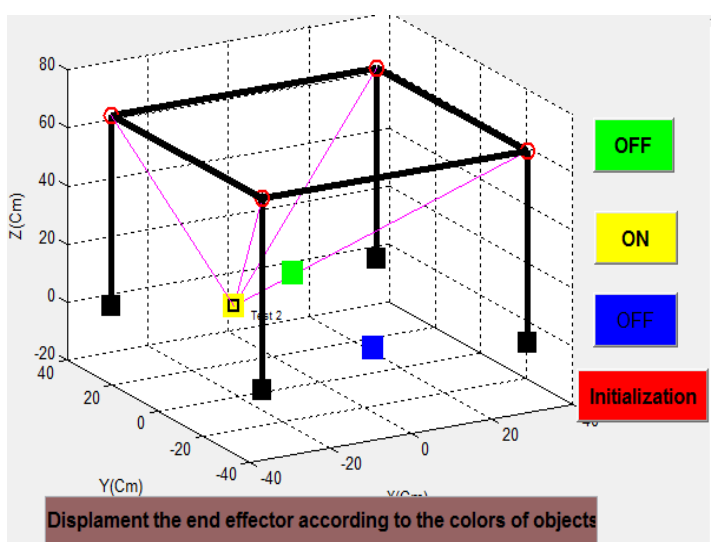

Figure 6. Displacement the end effector according to the yellow color

For more illustrate the role of our control, we simulate the continuance trajectories, Figure 8 shows that the desired and actual path for square trajectory. The actual path is in good agreement with the desired path. This shows the simulation and the control is very good results. 


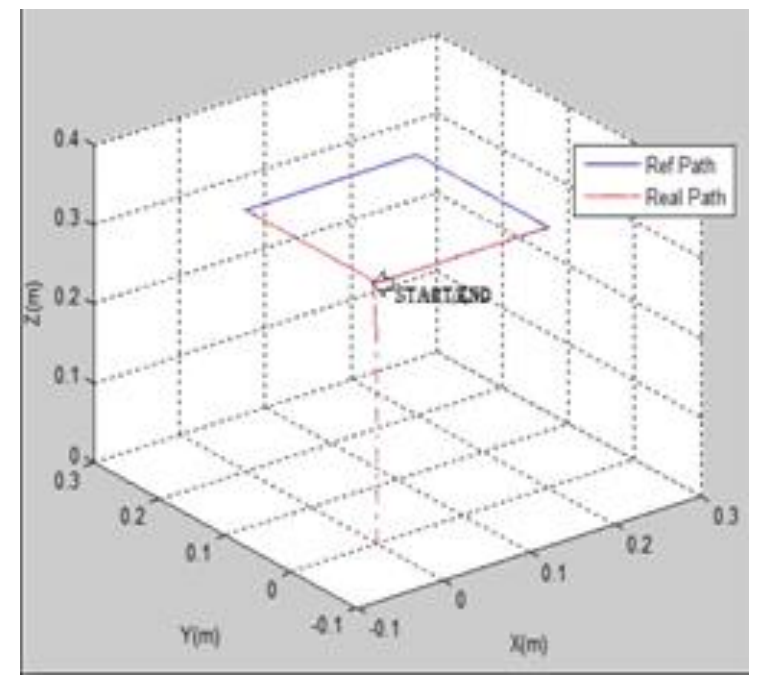

Figure 7. Plot the desired path and actual for square trajectory

\section{CONCLUSION}

This paper presented a simulation results for different tests of a novel 3D cable base robot with four cables, this last, we have designed as a control the generalize predictive mode. Then, we developed an user interface graphic with a simulation program to control the displacement of end effector based on: point to point command, according to the color of the objects and according to the a predefined point, we assume that , the tensions values are limited with tmin and tmax which always positive and the cables lengths do not exceed the workspace. In addition, we have presented some results for continuance trajectories. The simulation results have demonstrated the effectiveness and feasibility of the proposed control and suitable for improving the performance response.

\section{REFERENCES}

[1] 1. Giovanni Boschetti, G.Carbone and C.Passarini , "Cable Failure Operation Strategy for a Rehabilitation Cable-Driven Robot', Robotics , 2019, 8, 17.

[2] T. Bruckmann, M.Meik and P.Lemmen, 'Concept Studies of Automated Construction Using Cable-Driven Parallel Robots", In Cable-Driven Parallel Robots. Springer International Publishing, 2018.

[3] G.Mottola, C. Gosselin, and M. Carricato, 'Dynamically feasible motions of a class of purely-translational cablesuspended parallel robots"', Mechanism and Machine Theory, 2019. 132: p. 193-206.

[4] R.L. Williams IIJ,S. Albus and R.V. Bostelman, '3D CableBased Cartesian Metrology System'. Journal of Robotic Systems, 2004. 21(5): p. 237-257.

[5] M. Demirel, 'Design of hybrid cable-constrained parallel mechanisms for walking machines", Izmir Institute of Technology, 2018.
[6] B. Zi, J.L. Du, B.Y. Duan and H. Bao, ' Dynamic modeling and active control of a cable-suspended parallel robot', Mechatronics, 2008. 18(1): p. 1-12.

[7] Y.Tao, X. Xie and H. Xiong, 'An RBF-PD Control Method for Robot Grasping of Moving Object, in Transactions on Intelligent Welding Manufacturing',, Springer. 2019, p. 139-156.

[8] M.A. Khosravi, H.D. Taghirad, 'Dynamic modeling and control of parallel robots with elastic cables: singular perturbation approach', IEEE Transactions on Robotics, 2014. 30(3): p. 694704

[9] F.Inel, B. Bouchmal and L.Khochmane, 'Modeling and control of new model in a spatial coordinates -3D- for cable-based robot', World Journal of Engineering, 2015.Vol. 12 No. 2, pp. 189-200.

[10] R.L.Williams, P. Gallina, and J. Vadia, 'Planar Translational Cable-Direct-Driven Robots', Journal of Robotic Systems, 2003. 20(3): p. 107-120.

[11] A.Isidori,'Nonlinear control systems', Springer-Verlag London, 3rd edition, 1995,pp.293-297.

[12] R.Errouissi, M.Ouhrouche and W-H.Chen, ''Robust Nonlinear Generalized Predictive Control of a Permanent Magnet Synchronous Motor with an Anti-Windup Compensator', IEEE International Symposium on Industrial Electronics, 2010,pp. 